\title{
Position of Fingerprint Location based on Improved Universal Kriging Interpolation Method
}

\author{
Zhongwen Wang, Xin Ge, Ruizhen Duan*, and Mingshan Chi \\ Rongcheng College, Harbin University of Science and Technology, Rongcheng, 264300, China
}

\begin{abstract}
The accuracy of fingerprint databases is very important to position fingerprint locations. However, due to the measurement of the received signal strength cost and artificial constraints, the measured RSSI is limited, resulting in insufficient accuracy of fingerprint databases. Therefore, we adopt the spatial interpolation algorithm to obtain a more accurate location fingerprint database and innovatively propose an improved universal Kriging interpolation method, introducing variable parameters, overcoming the shortcomings of large measurement errors in the variation function, and generating an accurate location fingerprint database. The simulation results show that compared with the universal Kriging interpolation method and the inverse distance weighting method, the standard error of prediction is increased by $76.33 \%$ and $96.50 \%$, respectively.
\end{abstract}

Keywords: position fingerprint; universal Kriging interpolation; improved universal Kriging interpolation; Gaussian filtering; Gaussian function

(Submitted on February 20, 2019; Revised on March 5, 2019; Accepted on June 28, 2019)

() 2019 Totem Publisher, Inc. All rights reserved.

\section{Introduction}

At present, indoor location technology can generally be divided into approximation, geometry, and scene analysis [1-3] based on the received signal strength indicator (RSSI). The position fingerprint method is a scene analysis method. Because the algorithm can be pure software and can have a higher accuracy, this method has been widely used [4-5]. It is divided into two stages: the offline stage, which involves the wireless signal strength as the scene characteristic and constitutes the location of the finger-print database [6], and the online stage, which involves the use of matching algorithm real-time access to location information [7]. Most research has focused on the online matching algorithm to obtain higher positioning accuracy but ignored the offline stage of the collected signal value processing and extraction of positioning characteristics of the process [8]. The accuracy of the fingerprint database directly affects the accuracy of the positioning results to a large extent. The establishment of high-precision fingerprint databases is a prerequisite for highprecision positioning results [9-13].

In this paper, we innovatively use an improved universal Kriging interpolation method for the offline phase of processing. Since the continuity and variability of signal strength are very consistent with the characteristics of spatiotemporal random variables in spatial variability theory [14-15], spatial interpolation methods are used.

The core of the spatial interpolation algorithm is the variation function. The spatial correlation between the RSSI data of the measurement points is described by establishing the variation function model of the spatial statistics. We propose an improved universal Kriging interpolation method, which changes the variational function model to make it more suitable for the characteristics of spatial signals. The simulation results show that the improved algorithm is better than other algorithms, and the accuracy of the prediction is ensured while the number of training points is reduced. This method improves the accuracy of indoor positioning and reduces the economic cost of positioning [16].

\footnotetext{
* Corresponding author.

E-mail address: wangzhongwen1979@qq.com
} 


\section{Location Fingerprint Database Establishment}

\subsection{Application Principle of the Inverse Distance Weighting Method}

The inverse distance weighting method (IDW) is one of the most commonly used spatial interpolation methods [17-18]. It is based on the similarity principle, measured by the distance between the known and unknown points. The formula is:

$$
\left\{\begin{array}{l}
Z\left(x_{0}\right)=\sum_{i=1}^{n} \lambda_{i} Z\left(x_{i}\right) \\
\lambda_{i}=d_{i 0}^{-p} / \sum_{i=1}^{n} d_{i 0}^{-p}
\end{array}\right.
$$

In the formula, $\lambda_{i}$ is the weight of the known point (sample point) to the point to be measured, $d_{i 0}$ is the distance between the sample point and the point to be measured, and the default value of $p$ is 2 [19]. The universal Kriging interpolation method and the inverse distance weighting (IDW) method follow the principle that the weight of the sample points is closer to the distance to be measured. However, the universal Kriging interpolation method also considers the spatial correlation of each point and the spatial distribution of the sample points.

\subsection{Application Principle of the Universal Kriging Interpolation Method}

The weighted value of the universal Kriging interpolation method may be less than 0 or greater than 1 , so it can be less than the minimum sample value or greater than the maximum sample value, which is more in line with the real situation. This is one of the advantages of the universal Kriging method.

In the actual positioning applications, the propagation of the signal is attenuated. Each sampling point $\operatorname{RSSI}_{i}(i=1,2, \cdots, n)$ shows the change attribute of the signal in the environment and position, which contains the propagation attenuation characteristic of the signal in the environment because of the distance change and the variation characteristic under the external space interference in the complex indoor space [20]. Let $Z(l)=R S S I_{i}$, and then the model is as follows:

$$
Z(l)=M(l)+R(l)
$$

In the formula, $l$ is the position coordinates and $M(l)$ is the drift, reflecting the RSSI overall trend. $R(l)$ is a random part (residual), which reflects the random noise contained in the RSSI sample value and the variation of the sampling point in space.

\subsubsection{Determining the Drift}

Under normal circumstances, the spatial signal distribution has a certain trend of regular changes. According to the definition of drift and residuals in the universal Kriging interpolation method, Equation (2) is given.

$$
E[Z(l)]=M(l), E[R(l)]=0
$$

From Equation (3), we know that the residual $R(l)$ satisfies the second order stationary hypothesis. If $M(l)$ is known, we can subtract $M(l)$ from the original RSSI value and then use the ordinary Kriging method to estimate the $R(l)$. Thus, the drift function $M(l)$ of a certain point is considered as a polynomial combination based on the position information of the point, and the deterministic partial drift function can be expressed as a polynomial. They are calculated in Equations (4) and (5).

$$
M(x, y)=\sum_{k=0}^{K} a_{k} p_{k}(x, y)
$$




$$
Z(x, y)=\sum_{k=0}^{K} a_{k} p_{k}(x, y)+R(x, y)
$$

In the formula, $(x, y)$ is the position coordinate of the point, $a_{k}$ is a set of constant parameters, $K$ is the drift coefficient dimension, and $p_{k}(x, y)$ is the spatial function of the position.

In this paper, the location of the fingerprint database is a two-dimensional space, and secondary drift is selected [21-22], as shown in Equation (6).

$$
M(x, y)=a_{0}+a_{1} x+a_{2} y+a_{3} x^{2}+a_{4} y^{2}+a_{5} x y
$$

Then, the drift polynomial coefficients are estimated by combining the original data and applying the multiple stepwise regression method [23-25].

\subsubsection{Determining Residual}

After removing the RSSI drift trend, the residual $R(l)$ satisfies the second order stationary hypothesis. According to the general principle of universal Kriging interpolation method, the interpolation formula for predicting the residuals can be estimated in Equation (7).

$$
\hat{R}\left(l_{0}\right)=\sum_{i=1}^{n} \lambda_{i} R\left(l_{i}\right)
$$

In the formula, $\lambda_{i}(i=1,2, \cdots, n)$ is the required weight coefficient, and $\sum_{i=1}^{n} \lambda_{i}=1$. The position of the sampling point is $l_{i}(1 \leq i \leq n)$, and $R\left(l_{i}\right)$ is the residual of the corresponding sampled signal strength. $\hat{R}\left(l_{0}\right)$ is an unbiased estimate of $R\left(l_{0}\right)$. $R(l)$ satisfies the second order stationary hypothesis and obtains the variance of the estimated variance in Equation (8).

$$
\begin{aligned}
\sigma^{2} & =E\left[\hat{R}\left(l_{0}\right)-R\left(l_{0}\right)\right]^{2}-\left\{E\left[\hat{R}\left(l_{0}\right)-R\left(l_{0}\right)\right]\right\}^{2} \\
& =\operatorname{Cov}\left(l_{0}, l_{0}\right)-2 \sum_{j=1}^{n} \lambda_{j} \operatorname{Cov}\left(l_{0}, l_{j}\right)+\sum_{i=1}^{n} \sum_{j=1}^{n} \lambda_{i} \lambda_{j} \operatorname{Cov}\left(l_{i}, l_{j}\right)
\end{aligned}
$$

In the formula, $\operatorname{Cov}\left(l_{i}, l_{j}\right)$ is the covariance function of $R\left(l_{i}\right)$ and $R\left(l_{j}\right)$. In the unbiased estimation condition, the estimated variance is minimized, and it is calculated in Equation (9).

$$
\left.\min \left\{\operatorname{Var}\left[\hat{R}\left(l_{0}\right)-R\left(l_{0}\right)\right]\right\}-2 \xi \sum_{i=1}^{n}\left(\lambda_{i}-1\right)\right\}
$$

In the formula, $\xi$ is the Lagrangian multiplier. After solving the equation, we obtain the weight coefficient equation group, as shown in Equation (10).

$$
\left\{\begin{array}{l}
\sum_{i=1}^{n} \lambda_{i} \operatorname{Cov}\left(l_{i}, l_{j}\right)-\xi=\operatorname{Cov}\left(l_{0}, l_{j}\right), 1 \leq j \leq n \\
\sum_{i=1}^{n} \lambda_{i}=1
\end{array}\right.
$$

\subsubsection{Estimation of Variation Function}

When the Lagrangian function is used to find the optimal solution, the covariance in the matrix cannot be obtained directly. Therefore, it is the key to solve the problem by transforming the covariance into the variation of the spatial variable and using the variation function. The variation function is defined in Equation (11). 


$$
\gamma\left(l_{i}, l_{j}\right)=\frac{1}{2} E\left[R\left(l_{i}\right)-R\left(l_{j}\right)\right]^{2}
$$

Under the second order stationary hypothesis, the autocorrelation between $R(l)$ and $R(l+h)$ does not depend on the actual position $l$; it only depends on the displacement between the two parts, so the following Equation (12) can be obtained. Thus, an estimate of the expression of the variance function can be deduced [24], as shown in Equation (13).

$$
\begin{gathered}
E[R(l+h)-R(l)]=0 \\
\hat{\gamma}(h)=\frac{1}{2 N(h)} \sum_{i=1}^{N(h)}[R(l+h)-R(l)]^{2}
\end{gathered}
$$

In the formula, $h$ is the separation distance between the variables, $N(h)$ is the logarithm of the sampling point satisfying the separation distance $h$, and $R(l), R(l+h)$ are the sampling point signal strength values at position $l$ and position $l+h$.

\subsection{Improved Universal Kriging Interpolation Method}

RSSI has a continuous attribute, so the variable function is usually the selected Gaussian model. Although the Gaussian function is the most common case of the variation function, the block gold value of the Gaussian function is too large. That is affected by the greater degree of uncertainty, and the forecast process will strengthen the "filter effect" [26-29]. This may make the negative weight too high. Therefore, it can result in abnormal estimation results [30-31].

Considering the variation function as long as the positive function is satisfied, we can conclude that the function value is valid. In this paper, we introduce the variable parameter $\beta$, instead of the parameter value in the original Gaussian model. The improved version is shown in Equation (14).

$$
r(h)=\left\{\begin{array}{ccrl}
0, & h & =0 \\
C_{0}+C\left[1-e^{-(h / a)^{\beta}}\right], & 0<h & <\sqrt[\beta]{3} a \\
C_{0}+C, & h & \geq \sqrt[\beta]{3} a
\end{array}\right.
$$

In Equation (14), $C$ represents the arch height (partial still value) and $C_{0}$ represents the block gold value. $C_{0}$ reflects the random size of the region variable RSSI, which reflects the RSSI sample values containing random noise and sampling points in the spatial variation characteristics. It also reflects the extent of the impact of uncertainty factors. $C_{0}+C$ reflects the variation of regional variables. $a$ represents the range (maximum distance of spatial correlation). $h$ represents the separation distance.

When taking different $\beta$ values, the model parameters will change, and the prediction error will be different. As the value of $\beta$ increases, the RMSE (root mean square error) and block gold value of the two parameters first decrease and then increase. When the RMSE reaches its minimum value, $\beta$ is located between 0.1-0.6, and at this point the block gold value tends to 0 . Therefore, the variation function model conforms to the principle that the prediction error and the block gold value are smaller.

\section{Simulation and Analysis}

In order to verify the performance of the location fingerprint database established by the improved universal Kriging interpolation method, we select a $20 \times 15 \mathrm{~m}^{2}$ room, and the WLAN router location is shown in Figure 1 .

We take $18 \times 14 \mathrm{~m}^{2}$ in this area as the positioning area. It is separated by two meters. Figure 1 shows the measurement point. The RSSI is collected by wifi signal acquisition software, assuming that the quantized value of the received signal strength indicator (wifi analyzer) can truly reflect the actual received signal strength. We use ArcGIS software to simulate the universal Kriging interpolation method. 


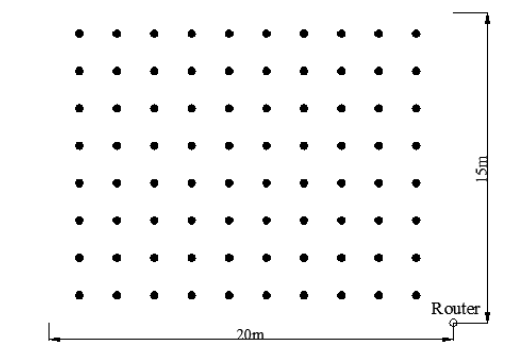

Figure 1. Experimental positioning area map

\subsection{RSSI Attribute Value Statistical Analysis}

Firstly, we collect the received signal intensity (RSSI) values of 80 sampling points in the positioning area. Then, we collect 100 times for each sampling point and perform the numerical analysis of the RSSI values of each point after Gaussian filtering, as shown in Table 1.

Table 1. Sampling point RSSI attribute value analysis statistics table

\begin{tabular}{cc}
\hline Statistics & The value of the statistic \\
\hline Number of samples & 80 \\
Sample mean/dbm & -70.338 \\
Maximum value/dbm & -54 \\
Minimum value/dbm & -87 \\
Median/dbm & -71 \\
Standard deviation/dbm & 7.619 \\
Skewness & 0.035059 \\
Kurtosis & 2.7811 \\
\hline
\end{tabular}

Then, we analyze the trend, and the trend analysis is shown in Figure 2. We can see from the 3D analysis that the signal strength in both directions on the projection shows a downward trend. This is consistent with the objective law of using the universal Kriging interpolation method. It can be seen from Figure 3 that the data of the normal distribution QQ graph is similar to the normal distribution. This shows that the sampling data can represent the distribution of universal data.

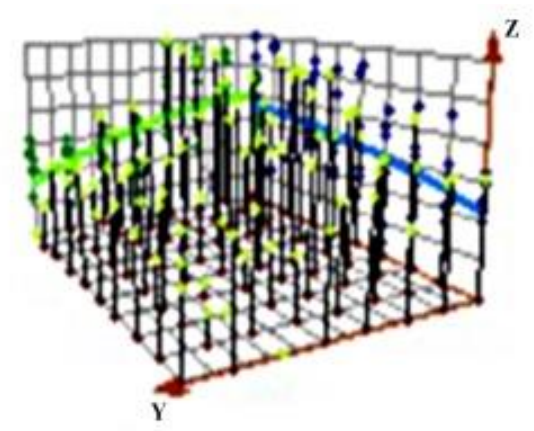

Figure 2. Sampling data trend analysis drawing

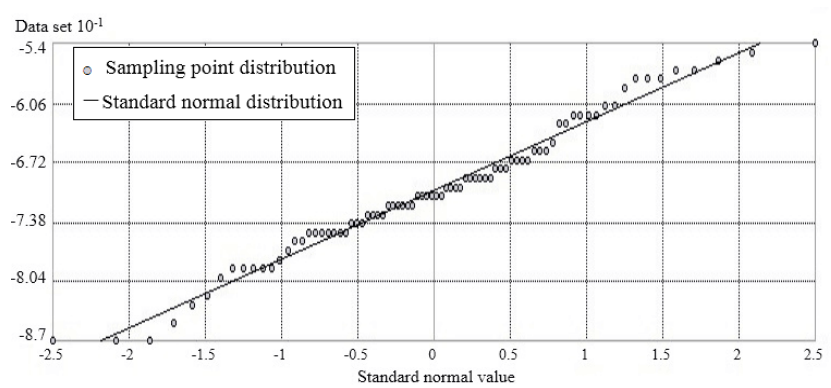

Figure 3. Sampling data normal distribution QQ graph

From Table 1 and Figures 2-3, the RSSI value approximates the normal distribution. According to the processed data, we can obtain the regional RSSI distribution, as shown in Figure 4. Then, we deal with it using the universal Kriging interpolation method. 


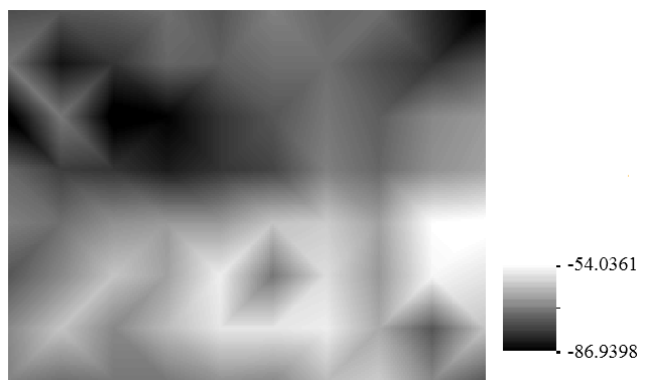

Figure 4. Distribution of the RSSI values for the locating area

\subsection{RSSI Spatial Variability Analysis}

This section is the simulation of the improved variation function model, and the variation function is the core of the universal Kriging interpolation method. The theoretical part of the article has already described how to improve the variation function in the process of universal Kriging interpolation in order to better apply it to the problem of spatial signals.

We must calculate the difference in the RSSI values between any two measurement points in the locating area in order to describe the spatial variability of the RSSI. Therefore, in this paper, we adopt the improved Gaussian function. The Gaussian function model is suitable for the interpolation of space signals. Figure 5 shows the improved Gaussian function fitting image.

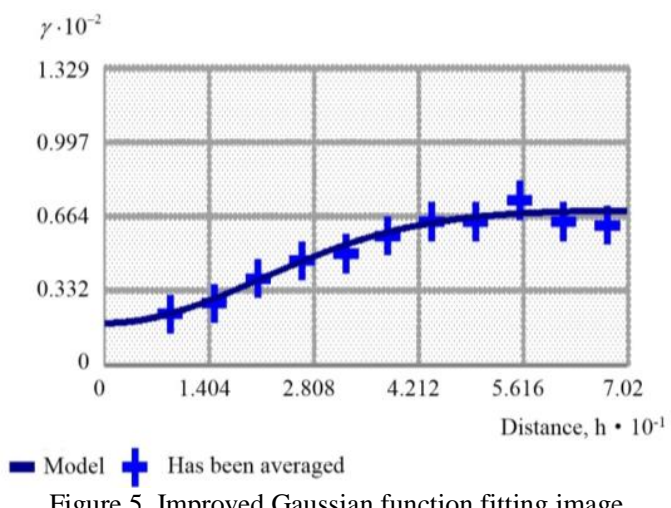

\subsection{RSSI Spatial Variability Analysis Comparison of Forecast Results}

\subsubsection{Comparison of Different Numbers of Training Points}

This article has a total of 80 measurement points' RSSI values. In the random sampling, the training points at 25, 30, 35, 40, 45, and 60 are measured respectively. Then, the RSSI values of the training samples are predicted using the improved universal Kriging interpolation method (IUKI), the universal Kriging interpolation method (UKI), and the inverse distance weighted interpolation method (IDW). The predicted values are compared with the actual measured values of the training samples, and the prediction accuracy of the three methods under different training samples is obtained. The results are shown in Figure 6.

In Figure 6, according to the overall trend of the three lines (three interpolation methods), it can be seen that the average standard error decreases as the number of training points increases. The more training points that are chosen, the higher the prediction accuracy and fingerprint density. However, the increase in training points (known RSSI numerical points) means that the acquisition of RSSI values requires more manpower and material resources, and positioning costs increase.

Therefore, it is necessary to reduce the number of training points as much as possible and maintain the accuracy of the prediction. It can be seen from Figure 6 that regardless of the number of training points, the IDW average standard error is relatively large. However, as the number of training points increases, the average standard error of IDW decreases rapidly and the prediction accuracy is improved. Therefore, IDW is more suitable for use in the case of a large number of training points. 


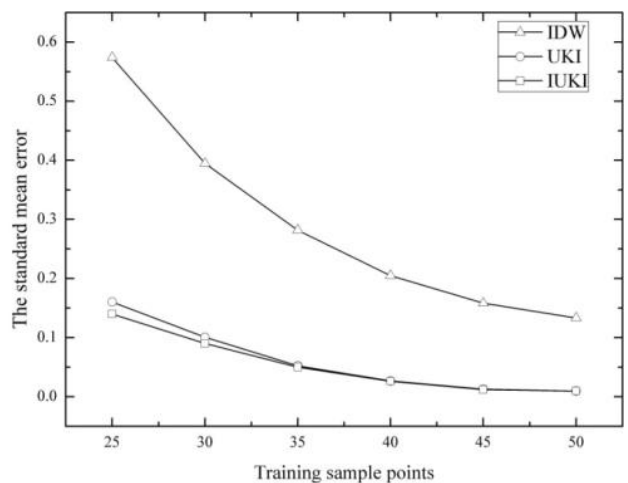

Figure 6. The degree of change in the average value standard error of different training samples

The IUKI and the UKI have little difference in the prediction accuracy after 35 training points, but the previous prediction accuracy is different. It can be seen from Figure 6 that under the premise of a small number of training points, the IUKI has higher prediction accuracy than the UKI. For the IUKI, when the training points of the origin are 50\%, the average standard error is $0.02672774 \mathrm{dbm}$. When the training points are $62 \%$, the average standard error is $0.0095136 \mathrm{dbm}$. This shows that the work is reduced compared to $100 \%$ point-by-point sampling, while ensuring the accuracy of prediction.

The interpolation of the RSSI in the positioning area with the IDW, the IUKI, and the UKI is shown in Figures 7-9, respectively.

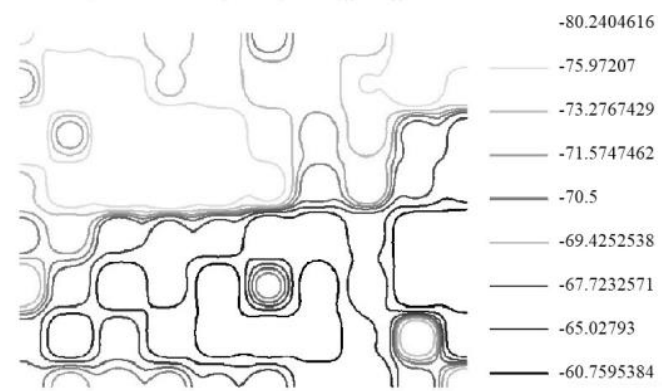

Figure 7. IDW RSSI interpolation prediction

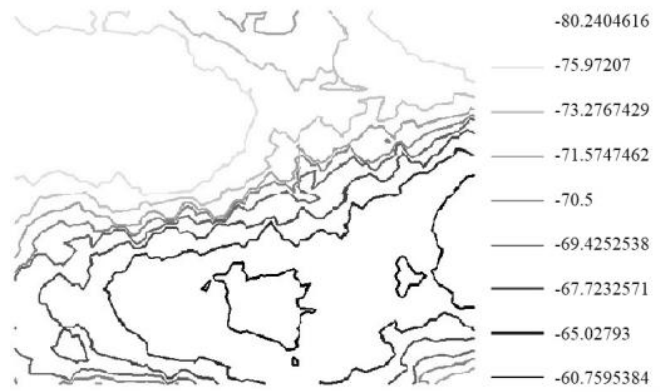

Figure 8. IUKI RSSI interpolation prediction

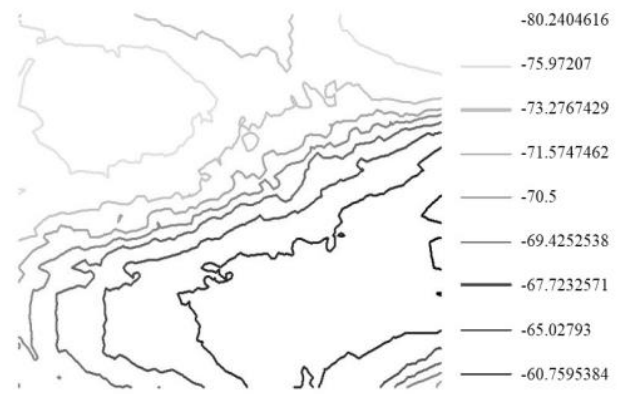

Figure 9. UKI RSSI interpolation prediction 


\subsubsection{Comparison of Prediction Accuracy}

We use the IUKI, UKI, and IDW methods to predict the unknown points and compare the predicted value of each point with the real value of the sampling points to draw the RSSI numerical comparison chart of each point, as shown in Figures 10-12.

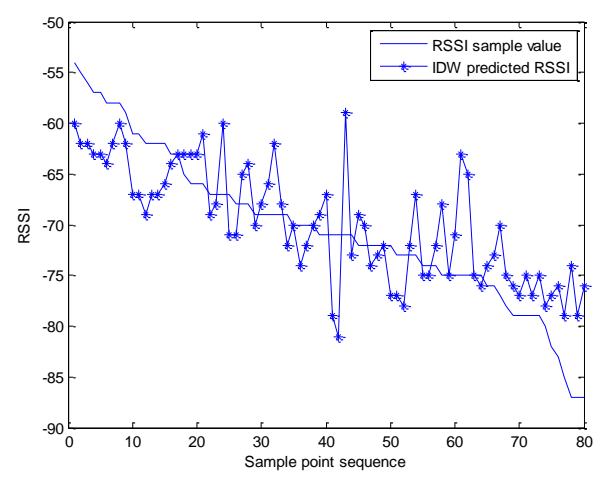

Figure 10. Comparison of IDW method's RSSI forecast

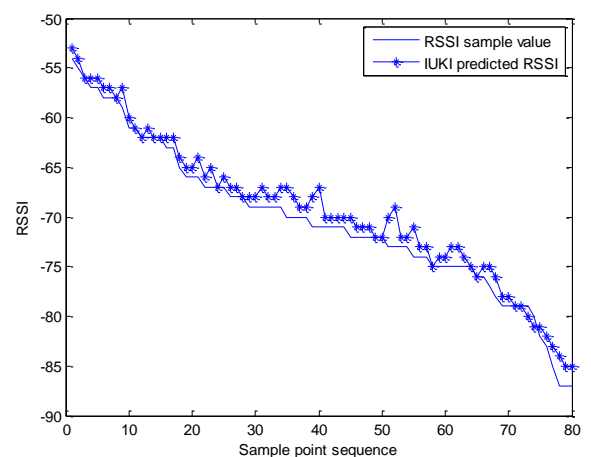

Figure 11. IUKI method's RSSI forecast

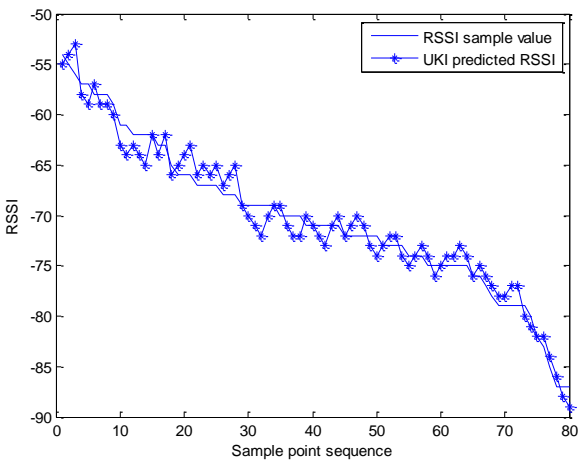

Figure 12. UKI method's RSSI forecast

According to Figures 10-12, the IUKI method is more suitable to establish the indoor positioning fingerprint database. It is clear that the predicted value of the IUKI is more similar to the true value, and the predicted data are more accurate.

For the validation of improved variation functions, the standard mean error and the standard root mean square error are generally used. As the arithmetic mean is affected by the size of the data, this paper examines the standard mean error. The value is close to 0 , and the deviation between prediction and measurement is small. The standard root mean square error is close to 1, and the standard average error is accurate.

Table 2 shows the standard mean error and standard root mean square error for the three interpolation methods $(80$ sample points are selected). From the numerical comparison, it is shown that the improved universal Kriging interpolation method improves the prediction accuracy. 
Table 2. Precision numerical table

\begin{tabular}{cccc}
\hline Interpolation methods & IDW & UKI & IUKI \\
\hline Standard mean error & 0.0774831 & 0.01144611 & 0.002709 \\
Standard root mean square error & 1.032675 & 0.98722 & 1.001273 \\
\hline
\end{tabular}

The UKI, IUKI, and IDW methods are used to predict the position points by weighting the actual measured values. However, the weight assigned by the IDW to the actual measured value depends entirely on the distance between the measured point and the predicted point, which is equivalent to the propagation model of the wireless signal in free space to describe the relationship between the intensity of the different position signals. This shows that the IDW does not apply to complex communication environments. The UKI and the IUKI are the combination of the variance of the spatial signal and the calculation of the weight coefficient, taking into account the fluctuation of the signal in the complex communication environment. The IUKI is more adaptable to the complex communication environment than the UKI, so more accurate prediction can be obtained.

\section{Conclusions}

We used the IUKI to perform two-dimensional interpolation of spatial signals. The spatial correlation between the RSSI data of the measurement points was described by the variation function model of the spatial statistics. After that, we measured the RSSI value of the unknown points by the IUKI method to generate a high-resolution location fingerprint database.

In this paper, the improved universal Kriging interpolation method was used to make innovative improvements to the key variable functions. On the basis of the Gaussian model, variable parameters were introduced to reduce the nugget value and make the change function model. It conformed to the principles of small prediction error and small block value. The simulation results showed that the proposed method can improve the accuracy of the location fingerprint database and reduce the number of sampling points. In the case of 80 sampling points, the standard average error of the UKI method was 0.002709 , which was $76.33 \%$ higher than UKI and $96.50 \%$ higher than IDW.

\section{Acknowledgments}

This work is supported by the Project of Shandong Province Higher Educational Science and Technology Program (No. J16LB59). In this paper, the first two authors, Ruizhen Duan and Xin Ge, conceived the idea, organized the work, designed the proposed algorithms, analyzed the results, and wrote the paper. Antonino Zhongwen Wang performed the experiments, and Mingshan Chi supervised the work. All the authors reviewed the writing of the paper, its structure, and its intellectual content.

\section{References}

1. Y. Xiong, D. X. Shi, and B. Ding, "Mobile Group Awareness Technology Research,” Journal of Computer, No. 41, pp. 1-8, 2014

2. B. Guo, Z. W. Yu, and X. S. Zhou, "From Participatory Sensing to Mobile Crowd," Sensing Computer Science, No. 6, pp. 593598,2014

3. H. Choi, Y. Koo, X. Lee, and S. Park, "Indoor Positioning System based on an Improved Weighted-Trilateration Algorithm with Fingerprinting Technique,” International Journal of Multimedia and Ubiquitous Engineering, Vol. 11, No. 9, pp. 165-176, 2016

4. F. Li, "ZigBee based on the Location of Fingerprint Positioning Technology," Harbin Institute of Technology, 2012

5. J. H. Han and X. Chen, "Construction Method of Location Fingerprint Database based on Gaussian Process Regression Modeling," International Journal of Database Theory and Application, Vol. 9, No. 10, pp. 141-150, 2016

6. J. Li, B. Zhang, and H. Liu, "An Indoor Hybrid Localization Approach based on Signal Propagation Model and Fingerprinting," International Journal of Smart Home, Vol. 7, No. 6, pp. 157-170, 2013

7. B. Cui and X. Zhao, "A Hybrid Indoor Location Method based on Propagation Model and Position Fingerprint," Research Report, No. 6, pp. 35-38+43, 2015

8. J. H. Shen, "Based on RSSI Fingerprint Localization Experiment and Its Optimization Algorithm," Zhejiang Signal Processing Society, No. 8, 2015

9. H. H. Zhang, D. L. Zhu, and W. M. Feng, "Improved Wi-Fi Location Fingerprinting Method for Indoor Personnel Location Tracking," Securing Science and Technology, No. 3, pp. 36-41, 2015

10. Z. J. Liu, W. G. Guan, H. L. Hua, and Z. H. Sun, "Study on Location Fingerprint Database based on Kriging Space Interpolation," Application Research of Computers, No. 10, pp. 3139-3142, 2016

11. A. G. Journel and M. E. Rossi, "When Do We Need a Trend Model in Kriging?" Mathematical Geology, Vol. 21, No. 7, pp. 715- 739, 1989

12. L. X. Song, C. B. Zhou, and H. Pei, "Location Fingerprint Positioning Technology using Bat Algorithm," International Journal of Security and Its Applications, Vol. 10, No. 9, pp. 99-108, 2016 
13. Y. X. Wang, G. Hua, and Y. Xu, "Wireless Positioning Algorithm based on RSS in Limited Space," International Journal of Signal Processing, Image Processing and Pattern Recognition, Vol. 9, No. 1, pp. 335-346, 2016

14. F. Li, T. C. Wang, and W. M. Tong, "A Method for Radio-Map Construction based on the Spatial Variability Theory," Journal of Harbin Engineering University, No. 6, pp. 715-719 + 740, 2012

15. S. T. Du, "Geostatistics Method Summary," Petroleum Geophysics, No. 4, pp. 31-38, 2004

16. J. L. Wang and D. M. Zhang, "Comparative Analysis of Spatial Data Interpolation Algorithm," Mining Survey, No. 2, pp. 55$57+4,2010$

17. M. E. J. Newman, "Modularity and Community Structure in Networks," Proceedings of the National Academy of Science, Vol. 103, No. 23, pp. 8577-8582, 2006

18. M. Chen, "Based on the Signal Strength of the WLAN Indoor Positioning Tracking System," Wuhan University, 2012

19. J. L. Wang and D. M. Zhang, "Comparative Analysis of Spatial Data Interpolation Algorithm,” Mining Survey, No. 2, pp. 55$57+4,2010$

20. F. Li, "Based on ZigBee Location Fingerprint Method Indoor Positioning Technology Research," Harbin Institute of Technology, 2012

21. G. Christakos, "Random Field Models in Earth Sciences," Academic Press, Inc California, 1992

22. D. Marcotte and M. David, "Trend Surface Analysis as a Special Case of IRF-k Kriging," Mathematical Geology, Vol. 20, No. 7, 1988

23. Y. M. Lu, T. X. Yue, C. F. Chen, Z. M. Fan, and Q. M. Wang, "Multi-Stepwise Regression Simulation of Total Solar Radiation in China," Journal of Remote Sensing, No. 5, pp. 852-864, 2010

24. G. L. Yang, G. M. Zhang, and S. X. Li, "Application of the Universal Kriging Interpolation Method in Geomagnetic Map," Journal of Chinese Inertial Technology, No. 2, pp. 162-166, 2008

25. Q. Sun, L. Xue, and Y. Y. Wang, "Kriging Parameter Estimation Method and its Application in Parameter Estimation Analysis," Rock and Soil Mechanics, Vol. 30, No. S2, pp. 371-373, 2009

26. R. D. Zhang, "Spatial Variability Theory and Application (in Chinese)," Beijing Science Press, pp. 13-37, 2005

27. Y. Lin, X. Zhu, Z. Zheng, Z. Dou, and R. Zhou, "The Individual Identification Method of Wireless Device based on Dimensionality Reduction and Machine Learning," Journal of Supercomputing, No. 5, pp. 1-18, 2017

28. Y. Lin, C. Wang, J. X. Wang, and Z. Dou, "A Novel Dynamic Spectrum Access Framework based on Reinforcement Learning for Cognitive Radio Sensor Networks," Sensors, Vol. 16, No. 10, pp. 1-22, 2016

29. Y. Lin, C. Wang, C. Ma, Z. Dou, and X. Ma, "A New Combination Method for Multisensor Conflict Information," Journal of Supercomputing, Vol. 72, No. 7, pp. 2874-2890, 2016

30. Q. Wu, Y. Li, and Y. Lin, "The Application of Nonlocal Total Variation in Image Denoising for Mobile Transmission," Multimedia Tools \& Applications, Vol. 76, No. 16, pp. 1-13, 2016

31. C. Shi, Z. Dou, Y. Lin, and W. Li, "Dynamic Threshold-Setting for RF-Powered Cognitive Radio Networks in Non-Gaussian Noise," Physical Communication, Vol. 27, No. 4, pp. 99-105, 2018 